\title{
P38/TRHr-Dependent Regulation of TPO in Thyroid Cells Contributes to the Hypothyroidism of Triclosan-Treated Rats
}

\author{
Pei Zhang ${ }^{a} \quad$ Min Yang ${ }^{b} \quad$ Li Zeng $^{b} \quad$ Changjiang Liu ${ }^{b}$ \\ aDepartment of Gastrointestinal Surgery, Union Hospital, Tongji Medical College, Huazhong University

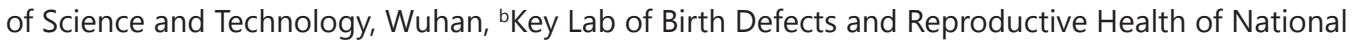 \\ Health and Family Planning Commission, Chongqing Population and Family Planning Science and \\ Technology Research Institute, Chongqing, China
}

\section{Key Words}

Triclosan • Hypothyroidism • P38/TRHr • TPO • Deiodinase $3 \cdot$ Hepatic enzymes

\begin{abstract}
Background/Aims: Triclosan, as an antimicrobial agent and a potential endocrine disruptor, has been used extensively in diverse products, resulting in widespread human exposure. In recent years, studies suggest that triclosan could disturb thyroid functions and decline thyroid hormones (THs). Methods: To verify our hypothesis that the MAPK pathway may function significantly in triclosan-induced hypothyroidism, Sprague-Dawley rats were gavaged with triclosan for 31 consecutive days; Nthy-ori 3-1 cells were treated with triclosan in the presence/ absence of NAC, inhibitors (SB203580 and SB202474), or TRHr siRNA. Tissues and/or cells were analyzed by several techniques including transmission electron microscopy, confocal laser scanning microscopy, gene silencing, western blot, and real-time PCR. Results: Triclosan led to histopathologic changes in the thyroid and decreases in triiodothyronine (T3) and thyroxine (T4). Triclosan stimulated ROS production and oxidative stress occurrence, thereby activating the p38 pathway in vivo and in vitro. Thyrotropin releasing hormone receptor (TRHr) was induced when the p38 pathway was activated, and was suppressed when that pathway was inhibited. Moreover, thyroid peroxidase (TPO) was restrained and modulated by the p38/TRHr pathway after triclosan treatment. Furthermore, deiodinase 3 (D3) and hepatic enzymes (Ugt2b1, CYP1a1, CYP1a2, CYP2b1, CYP3a1, and Sult1e1) were also induced by triclosan. Conclusion: Taken together, p38/TRHr-dependent regulation of TPO in thyroid cells contributes to the hypothyroidism of triclosan-treated rats.

(C) 2018 The Author(s)

Published by S. Karger AG, Basel
\end{abstract}

\section{Introduction}

Triclosan is a broad-spectrum, lipophilic, antimicrobial agent, which has been widely used in a variety of personal care products, household items, clinical settings, and medical 


\section{Cellular Physiology Cell Physiol Biochem 2018;45:1303-1315 \\ \begin{tabular}{ll|l} 
DOI: 10.1159/000487558 & (2018 The Author(s). Published by S. Karger AG, Basel \\
www.karger.com/cpb
\end{tabular} \\ Zhang et al.: Triclosan Causes Hypothyroidism via p38/TRHr-Regulated TPO}

devices. For the widespread use and bioaccumulation, triclosan is frequently detected in human blood plasma, breast milk, and urine $[1,2]$. Moreover, evidence indicates that triclosan has similar structures to thyroid hormones and both are halogenated biphenyl ethers [3]. Human exposures and the structural similarity have raised concerns about adverse effects of triclosan on thyroid homeostasis.

Thyroid hormones (THs) that are synthesized and released by the thyroid are essential for normal growth, development, and metabolism of organisms, as well as energy homeostasis. Even small changes or an imbalance in thyroid homeostasis may adversely affect human health. Especially for children, the disturbance of thyroid homeostasis during nervous system development would lead to irreversible mental retardation [4]. Therefore, disturbing factors of thyroid homeostasis are of great concern. In recent years, more studies have demonstrated that the thyroid is vulnerable to triclosan exposure, and triclosan could influence thyroid functions and lead to hypothyroidism in mammalian models [5-7]. Triclosan has also been shown to interfere with the thyroid axis in zebrafish [8] and frogs [9].

As relatively more attention has been focused on the interfering effects of triclosan on thyroid functions and TH homeostasis, some potential modes of action have been proposed to elucidate triclosan-induced hypothyroidism. A mode of action involved activations of pregnane X receptor (PXR) and constitutive androstane receptor (CAR), which induced activities of hepatic phases I and II enzymes and ultimately increased clearance of THs from the body [6]. Moreover, the study also reported that triclosan could accelerate the biliary excretion of THs through inducing TH sulfation [6]. Another mechanism proposed was related to TH receptors, including TR $\alpha$ and TR $\beta$. Exposure of Rana catesbeiana (North American bullfrog) to triclosan suppressed TR $\beta$ mRNA expressions in the tadpole tail fin, resulting in accelerated TH-dependent metamorphosis [10]. Additionally, some studies suggested that triclosan-induced hypothyroidism was the result of the suppression of $\mathrm{TH}$ biosynthesis. Triclosan was a non-competitive inhibitor of sodium/iodide symporter (NIS) and inhibited NIS-mediated iodide uptake in a concentration-dependent manner. Triclosan could also depress the activity of thyroid peroxidase (TPO) [11]. Furthermore, methyl triclosan was reported to affect TH-responsive gene transcripts in rat pituitary cells [12].

Thyroid hormone synthesis and secretion are regulated by the hypothalamic-pituitarythyroid (HPT) axis, and transduction of TH signaling is related to multiple signaling pathways such as the MAPK pathway. MAPKs that belong to a large family of serine-threonine kinases include three major subfamilies: ERK, JNK, and p38. The MAPK pathway is closely involved in cell cycle progression, cellular transformation, and differentiation of thyroid follicular epithelial cells. Moreover, it is proposed that the HPT axis may be an important target of the MAPK pathway [13], and our previous research also found that the JNK/MAPK pathway played significant roles in TH declines after PCB153 exposure [14]. However, whether the MAPK pathway functions significantly in triclosan-induced hypothyroidism is still unknown. Hereon, we hypothesized that the MAPK pathway might be associated with triclosan and reductions of THs. To validate our hypothesis, Sprague-Dawley rats and the human thyroid follicular epithelial cell line (Nthy-ori 3-1 cells) were used. The antioxidant, specific inhibitors of the MAPK pathway, and siRNAs were applied in the present study. The findings indicate that p38/TRHr-dependent regulation of TPO plays a vital role in triclosan-induced hypothyroidism, and our current study would contribute to a better understanding of the mechanisms by which triclosan interferes with thyroid homeostasis.

\section{Materials and Methods}

\section{Animals and treatments}

Male Sprague-Dawley rats (23 days old; $n=24$ ) were obtained from Daping Hospital Animal Laboratory (Chongqing, China) and randomly divided into four groups ( $n=6 /$ dose group) by counterbalancing body weights at the start of dosing. Animal facilities had an ambient temperature of $22 \pm 1{ }^{\circ} \mathrm{C}$, a relative humidity of $50 \pm 5 \%$, and a 12-h light-dark cycle. Food and water were provided ad libitum. Rats were dosed daily with triclosan (50, 100, and $200 \mathrm{mg} / \mathrm{kg} /$ day; CAS NO. 3380-34-5, DR Co., Germany) or corn oil (vehicle control) 


\section{Cellular Physiology Cell Physiol Biochem 2018;45:1303-1315 \begin{tabular}{l|l|l} 
and Biochemistry & DOI: 10.1159/000487558 & $\begin{array}{l}\text { C) } 2018 \text { The Author(s). Published by S. Karger AG, Basel } \\
\text { www.karger.com/cpb }\end{array}$ \\
\hline
\end{tabular}}

Zhang et al.: Triclosan Causes Hypothyroidism via p38/TRHr-Regulated TPO

via gavage for 31 days. The age of animals and duration and route of exposure were selected according to the recommendations of the US EPA Endocrine Disrupter Screening and Testing Advisory Committee; the doses were chosen based on the oral LD50 $(5000 \mathrm{mg} / \mathrm{kg})$ in rats. Dosing volumes were adjusted daily based on body weights of animals and all dosing was performed between 0800 and $1000 \mathrm{~h}$. All animal procedures were approved by the Research Ethics Committee of Chongqing Population and Family Planning Science and Technology Research Institute.

\section{Cells and treatments}

Nthy-ori 3-1 cells were treated with $10 \mu \mathrm{M}$ triclosan and collected at different time points $(0,1,3,6$, 12 , and $24 \mathrm{~h}$ ). Cells were pretreated with or without $1 \mu \mathrm{M}$ SB203580 (a specific inhibitor of p38 MAPK) or SB202474 (a negative control, an inactive analog of SB203580 with no p38-MAPK inhibitory potency) for $1 \mathrm{~h}$ before stimulation with triclosan. SB203580 and SB202474 were purchased from the Cell Signaling Technology, Inc. (MA, USA) and Gene Operation Inc. (Michigan, USA), respectively.

\section{Cell viability assay}

Nthy-ori 3-1 cells were treated with different concentrations of triclosan in 96-well plates for 24 or 48 $\mathrm{h}$, followed by incubation with $10 \mu \mathrm{l}$ of Cell Counting Kit-8 solution (Dojindo Laboratories, Japan) at $37{ }^{\circ} \mathrm{C}$ for $2 \mathrm{~h}$. Then the absorbance was measured at $450 \mathrm{~nm}$. Cell viability was calculated using baseline values of vehicle treated cells as a reference.

\section{Histopathologic evaluation}

Histopathologic changes in rat thyroids were detected by hematoxylin and eosin (HE) staining analysis and transmission electron microscope (TEM) analysis. Briefly, the thyroid was fixed in 4\% paraformaldehyde for $24 \mathrm{~h}$. Fixed thyroids were embedded, sliced and stained with HE. Then, histological alterations including numbers of follicular epithelial cells and diameters of thyroid follicular cavities were quantitatively evaluated using the image analysis software, Image-Pro Plus 6.0. Ultrastructural changes in the thyroid were assessed by TEM. Thyroids were diced after treatment in $2.5 \%$ glutaraldehyde at $4{ }^{\circ} \mathrm{C}$ for $24 \mathrm{~h}$. Then, slices of thyroids were postfixed in 1\% osmium tetroxide. FEI Tecnai $12 \mathrm{G} 2$ transmission electron microscope was used to examine sections.

\section{Hormone determination}

TT4, FT4, TT3, FT3, TSH, and TRH in plasma of the rat were detected using enzyme-linked immunoabsorbent assay kits (Assay Designs, Inc., USA). No significant cross-reactivity or interference was observed. Each sample was measured in duplicate.

\section{Malondialdehyde (MDA) measurement}

The MDA assay kit was used to analyze MDA level in plasma of the rat (Jiancheng Bioengineering Ltd., China). MDA level was analyzed measuring thiobarbituric-acid reacting substances at $532 \mathrm{~nm}$ and expressed as nmol MDA per milliliter plasma.

Immunohistochemical (IHC) and immunofluorescent (IF) staining analysis

After the antigen retrieval, sections of the pituitary, thyroid, and liver were deparaffinized, rehydrated and blocked in 3\% goat serum. For IHC analysis, the pituitary and thyroid sections were incubated with antiTRHr (Assay Biotechnology Inc., USA) overnight at $4{ }^{\circ} \mathrm{C}$, followed by treatments with biotinylated anti-rabbit IgG, peroxidase-conjugated streptavidin, diaminobenzidine substrate, and hematoxylin (Beyotime Biotech Inc., China). For IF analysis, the thyroid and liver sections were incubated with anti-p-p38 (Cell Signaling Technology Inc., USA) and anti-CYP1a1 (Abcam Inc., USA), respectively. Alexa Fluor 555-coupled goat antirabbit IgG and FITC-coupled goat anti-mouse IgG (Beyotime Biotech Inc., China) were applied for $30 \mathrm{~min}$ in dark. Nuclei were stained with DAPI for $5 \mathrm{~min}$. Treated cells were also incubated with anti-TRHr overnight at $4{ }^{\circ} \mathrm{C}$ for IF analysis.

\section{Confocal laser scanning microscopy (CLSM) analysis}

Nthy-ori 3-1 cells were fixed with 4\% paraformaldehyde for $20 \mathrm{~min}$ and dealt with normal goat serum for $30 \mathrm{~min}$. After incubation with anti-TPO (Abcam Inc., USA) overnight at $4{ }^{\circ} \mathrm{C}$, cells were treated with the secondary antibody and DAPI $(10 \mu \mathrm{g} / \mathrm{ml})$. Then cells were mounted with antifade polyvinylpyrrolidone mounting medium for CLSM analysis. 


\section{Cellular Physiology Cell Physiol Biochem 2018;45:1303-1315

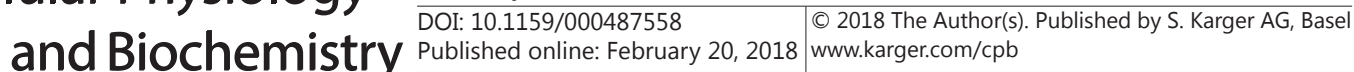

\section{ROS determination}

Nthy-ori 3-1 cells were treated with different concentrations of triclosan (0-10 $\mu \mathrm{M})$ for $24 \mathrm{~h}$ in the absence/presence of the antioxidant NAC (1 mM). Then cells were incubated with oxidant-sensitive probe DCFH-DA $(10 \mu \mathrm{M})$ at $37{ }^{\circ} \mathrm{C}$ for $20 \mathrm{~min}$. Rosup $(50 \mu \mathrm{g} / \mathrm{ml})$ was used as the positive control. Nthy-ori 3-1 cells were collected, resuspended, and seeded in a 96-well plate, followed by determinations using a fluorescent microplate reader (excitation wavelength, $488 \mathrm{~nm}$; emission wavelength $525 \mathrm{~nm}$ ).

\section{TRHr gene silencing}

Nthy-ori 3-1 cells grown to 60\% confluent were transfected with the TRHr siRNA (ID: s14395). The negative control siRNA (NC siRNA) was also purchased from the Life Technologies Corporation. siRNAs were dissolved in nuclease-free sterile water and diluted in Opti-MEM Medium to $400 \mathrm{nM}$. The siRNALipofectamine ${ }^{\circledR}$ RNAiMAX complex was prepared following manufacturer's protocols. Transfected cells were incubated at $37^{\circ} \mathrm{C}$ for $24 \mathrm{~h}$ and then the transfection efficiency was assessed using western blot analysis.

\section{Western blot}

Proteins were extracted and prepared from the rat hypothalamus, liver, and Nthy-ori 3-1 cells. Equal amounts $(20 \mu \mathrm{g} / \mathrm{lane})$ of protein were separated by SDS-PAGE and transferred electrophoretically onto a nitrocellulose membrane. After blocked by TBST containing 5\% non-fat milk for $1 \mathrm{~h}$, membranes were incubated with specific primary antibodies overnight at $4{ }^{\circ} \mathrm{C}$ and secondary antibodies for $1 \mathrm{~h}$ at $37{ }^{\circ} \mathrm{C}$. Ugt2b1, ERK, p-ERK, JNK, p-JNK, p38, p-p38, GAPDH, $\beta$-Actin, and $\beta$-Tubulin antibodies were purchased from the Cell Signaling Technology Inc. (USA). NIS, TPO, Tg, Sult2a1, TR $\alpha 1$, TR $\beta 1$, TSHr, and D2 antibodies were obtained from the Abcam Inc. (USA). CYP2b1 and Sult1e1 antibodies were bought from the Santa Cruz Biotechnology Inc. (USA). D1 and D3 antibodies were gotten from the Proteintech Group (China) and CloudClone Corp. (USA), respectively. TTR antibody was purchased from the Bioworld Technology Inc. (China). TRHr antibody was obtained from the Assay Biotechnology Inc. (USA).

\section{Real-time PCR}

After preparations of total RNA and cDNA, RT-PCR was performed using an ABI PRISM $^{\circledR}$ 7900HT Sequence Detection System. Thermal cycling conditions were as follows: UDG incubation at $50{ }^{\circ} \mathrm{C}$ for $2 \mathrm{~min}$ and initial denaturation at $95^{\circ} \mathrm{C}$ for $2 \mathrm{~min}$, followed by 40 cycles $\left(95{ }^{\circ} \mathrm{C}\right.$ for $15 \mathrm{sec}$ and $60{ }^{\circ} \mathrm{C}$ for $1 \mathrm{~min}$ ). Primer sequences were from the GenBank (Table 1).

\section{Data analysis}

Data were represented as the mean \pm SD. Statistical analyses of results were conducted by one-way ANOVA following appropriate transformations to the normal distribution and equal variance where necessary. Mean values were compared by Least-Significant Difference using SPSS. Differences were considered significant at $P<0.05$.

\section{Results}

Triclosan elevated liver weights of treated rats

No clinical signs of toxicity were observed in rats after triclosan treatment. Triclosan did not significantly affect the body weight and weight gain. Animals
Table 1. The primer sequences used in the present study

\begin{tabular}{|c|c|c|c|}
\hline Primer & Type & Primer Sequence & GenBank \\
\hline \multirow[t]{2}{*}{ CAT } & Forward & TGGCTATGGCTCACACACCTTC & NM_012520.2 \\
\hline & Reverse & GAGGCCATAATCCGGGTCTTC & \\
\hline \multirow[t]{2}{*}{ GPx1 } & Forward & AGGAGAATGGCAAGAATGAAGAGA & NM_030826.4 \\
\hline & Reverse & GGAAGGTAAAGAGCGGGTGAG & \\
\hline \multirow[t]{2}{*}{ GPx3 } & Forward & GGGCTTTGTGCCTAATTTCCAG & NM_022525.4 \\
\hline & Reverse & TTCTCAAAGTTCCAGCGGATGTC & \\
\hline \multirow[t]{2}{*}{ SOD1 } & Forward & AGCATGGGTTCCATGTCCATC & NM_017050.1 \\
\hline & Reverse & AGCCACATTGCCCAGGTCTC & \\
\hline \multirow[t]{2}{*}{ SOD2 } & Forward & TTCTGGACAAACCTGAGCCCTAA & NM_017051.2 \\
\hline & Reverse & GAACCTTGGACTCCCACAGACAC & \\
\hline \multirow[t]{2}{*}{ SOD3 } & Forward & GGGTCTGTCCTGTACTTCACCAGAG & NM_012880.1 \\
\hline & Reverse & CTGACATGGTCCAGGTGACAGAG & \\
\hline \multirow[t]{2}{*}{ Ugt1a1 } & Forward & TTGGTGGGATAAACTGCCTTCA & NM_012683.2 \\
\hline & Reverse & GAATTCTGCCCAAAGCCTCA & \\
\hline \multirow[t]{2}{*}{ Ugt1a6 } & Forward & CAGTCATGCCCAACATGATCTTC & NM_001039691.2 \\
\hline & Reverse & TCTCCGGAGGCGTTGACATA & \\
\hline \multirow[t]{2}{*}{ Ugt2b1 } & Forward & ACTTTAGACCTGGAGCCTGTGGAA & NM_173295.1 \\
\hline & Reverse & CTTGGTAGGCTCAATGAGAATGGAA & \\
\hline \multirow[t]{2}{*}{ CYP1a1 } & Forward & ATGAGTTTGGGGAGGTTACTGGT & NM_012540.2 \\
\hline & Reverse & ACTTCTTATTCAAGTCCTTGAAGGCA & \\
\hline \multirow[t]{2}{*}{ CYP1a2 } & Forward & AGTCCAGGAACACTATCAAGACTTCA & NM_012541.3 \\
\hline & Reverse & AGGGATGAGACCACCGTTGTC & \\
\hline \multirow[t]{2}{*}{ CYP2b1 } & Forward & TGAGAACCTCATGATCTCCCTGC & NM_001134844.1 \\
\hline & Reverse & AGGAAACCATAGCGGAGTGTGG & \\
\hline \multirow[t]{2}{*}{ CYP3a1 } & Forward & CTCTTCACCGTGATCCACAGCACT & NM_013105.2 \\
\hline & Reverse & ATGCTGCCCTTGTTCTCCTTGC & \\
\hline \multirow[t]{2}{*}{ Sult1a1 } & Forward & TTCGCAACGCCTACACAAAGA & NM_031834.1 \\
\hline & Reverse & TCACATGCACTAGCGGTGGAC & \\
\hline \multirow[t]{2}{*}{ Sult1b1 } & Forward & GCCCACAGAAATAATGGATCACAG & NM_022513.2 \\
\hline & Reverse & GTGCAGAACTCAAGTGTTGTTCCAG & \\
\hline \multirow[t]{2}{*}{ Sult1e1 } & Forward & GTGGTGCAATTTGAAGTGAACTGA & NM_012883.1 \\
\hline & Reverse & ATCTGGCCTTGCCAAGAATG & \\
\hline \multirow[t]{2}{*}{$\beta$-Actin } & Forward & GGAGATTACTGCCCTGGCTCCTA & NM_031144.2 \\
\hline & Reverse & GACTCATCGTACTCCTGCTTGCTG & \\
\hline
\end{tabular}


had normal activities during this period. However, the liver weight of treated rats exhibited a dose-dependent increase $(P=0.048$; Table 2$)$.

\section{Triclosan reduced TH levels}

THs in plasma of rats displayed a dose-dependent decrease after triclosan exposure, with the maximum effect observed in the high-dose group. Compared with the control, TT4, FT4, TT3, and FT3 levels in the $200 \mathrm{mg} / \mathrm{kg} / \mathrm{d}$ group were declined by $23.1 \%(P=0.002)$, $20.5 \%(P<0.001), 22.0 \%(P=0.001)$, and 19.1\% $(P=0.003)$, respectively. However, TSH and TRH levels were not significantly affected by triclosan relative to the control (Fig. 1A).

\section{Triclosan increased protein levels of TRß1 and TRHr}

Protein levels of TR $\beta 1$ and TRHr displayed increasing trends in the hypothalamus after triclosan treatment. Compared with the control, TR $\beta 1$ and TRHr in the high-dose group were elevated by 55.1\% $(P=0.003)$ and $49.0 \%(P<0.001)$, respectively. IHC analysis of TRHr protein expression also demonstrated the dose-dependent increasing trend in rat pituitary and thyroid. However, in rat hypothalamus there were no significant changes in TR $\alpha 1$ and TSHr protein expressions after triclosan treatment (Fig. 1B).

\section{Triclosan induced oxidative stress and ROS production}

After triclosan exposure, mRNA expressions of antioxidant enzymes in rat hypothalamus showed a decreasing trend, whereas MDA was accumulated. GPx3 (extracellular or plasmatic GPx), CAT, and SOD2 in the high-dose group were reduced by $41.9 \%(P=0.003), 33.9 \%$ $(P<0.001)$, and $23.3 \%$ $(P=0.007)$, respectively. Contrarily, MDA level was elevated by $63.7 \%$ over the control $(P=0.002$; Fig. 2A). Triclosan also stimulated ROS production in Nthy-ori 3-1 cells. After treatment with $10 \mu \mathrm{M}$ triclosan,

Table 2. Triclosan increased the liver weight of rats. Note. Values were reported as mean \pm SD. Relative liver weight is $\%$ body weight; * A difference at $\mathrm{P}<0.05$ was considered statistically significant

\begin{tabular}{cccc}
\hline Triclosan $(\mathrm{mg} / \mathrm{kg} / \mathrm{d})$ & Body wt $(\mathrm{g})$ & Body wt gain $(\mathrm{g})$ & Relative liver wt (\%) \\
\hline 0 & $264.57 \pm 29.1$ & $205.11 \pm 25.3$ & $3.56 \pm 0.3$ \\
50 & $256.80 \pm 18.0$ & $196.93 \pm 14.0$ & $3.71 \pm 0.2$ \\
100 & $255.71 \pm 26.9$ & $197.40 \pm 22.4$ & $3.86 \pm 0.3$ \\
200 & $246.60 \pm 20.1$ & $189.83 \pm 17.5$ & $4.04 \pm 0.4^{*}$
\end{tabular}

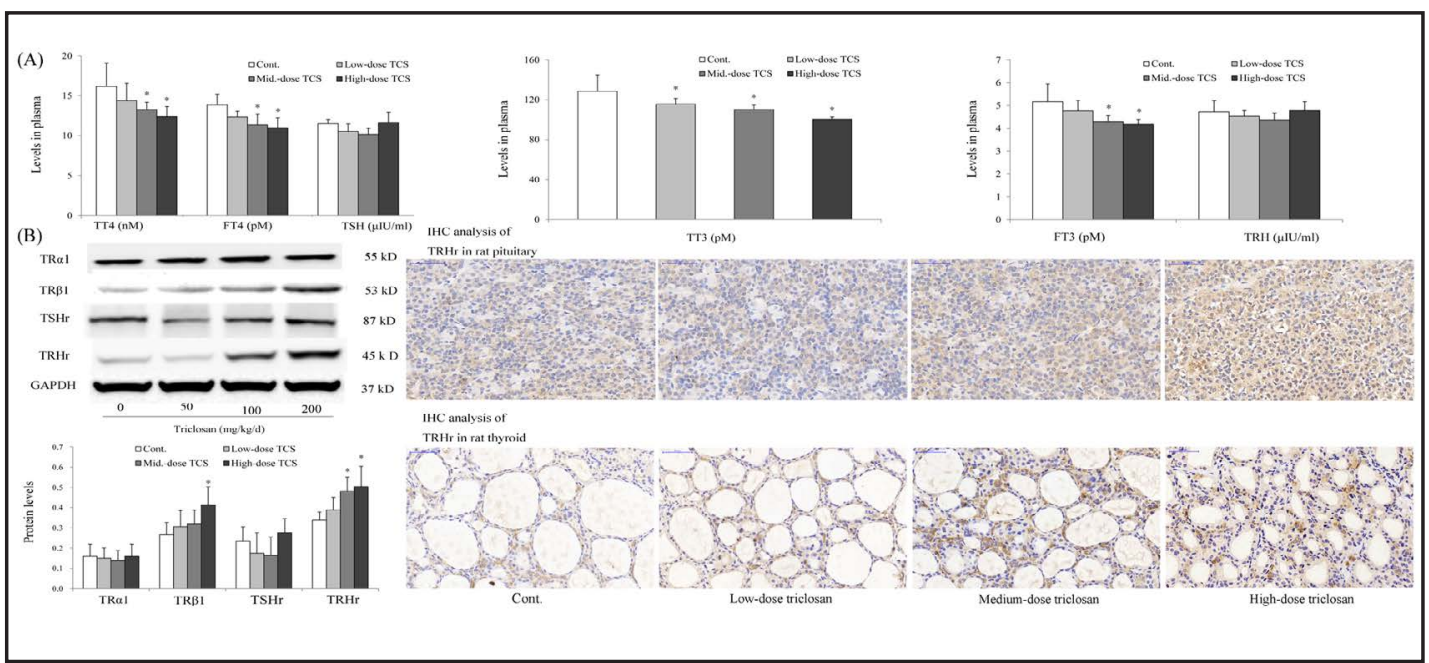

Fig. 1. Effects of triclosan on thyroid hormones and thyroid hormone-related receptors. (A) Triclosan reduced TH levels (TT4, FT4, TT3, and FT3) in rat plasma. (B) Triclosan upregulated protein expressions of TRß1 and TRHr in rat hypothalamus. IHC analysis exhibited the similar increasing trend of TRHr in rat pituitary and thyroid, the brown indicating positive expressions. Magnification: $\times 400 .{ }^{*} \mathrm{P}<0.05$, compared with the control. 
ROS level was increased by $49.5 \%$ over the control $(P<0.001)$. However, NAC effectively antagonized ROS production, which was downregulated by $41.7 \%$ compared to $10 \mu \mathrm{M}$ triclosan group $(P<0.001 ;$ Fig. 2B).

\section{Triclosan stimulated the protein expression of deiodinase 3}

Deiodinase 3 (D3) protein expression in rat liver was induced and that in the high-dose group had an $84.1 \%$ upregulation over the control $(P<0.001)$. However, D1 and D2 protein expressions in the liver were not affected after triclosan exposure (Fig. 2C). Moreover, protein expressions of deiodinases displayed the similar changing characteristics in the hypothalamus. D3 protein expression in the high-dose group was significantly higher than that in the control $(P=0.014)$, whereas no significant alterations in D1 and D2 protein expressions were observed (Fig. 2D). Triclosan also did not influence the transport of THs, and no significant alterations of TTR protein level in rat liver were observed (Fig. 2C).

\section{Triclosan inhibited TPO protein expression}

TPO protein level in rat liver was suppressed after triclosan treatment, with the maximum effect observed in the $200 \mathrm{mg} / \mathrm{kg} / \mathrm{d}$ group. TPO protein expression in the highdose group was downregulated by $29.5 \%$ compared with the control $(P=0.011)$. However, protein expressions of NIS and Tg in rat liver were not significantly affected by triclosan (Fig. 2E).

\section{Triclosan induced expressions of hepatic enzymes}

Triclosan induced expressions of hepatic enzymes in rat liver, including Ugts, CYPs, and Sults. Though triclosan did not affect mRNA levels of Ugt1a1 and Ugt1a6, gene and protein expressions of Ugt2b1 were both upregulated. In the high-dose group, Ugt2b1

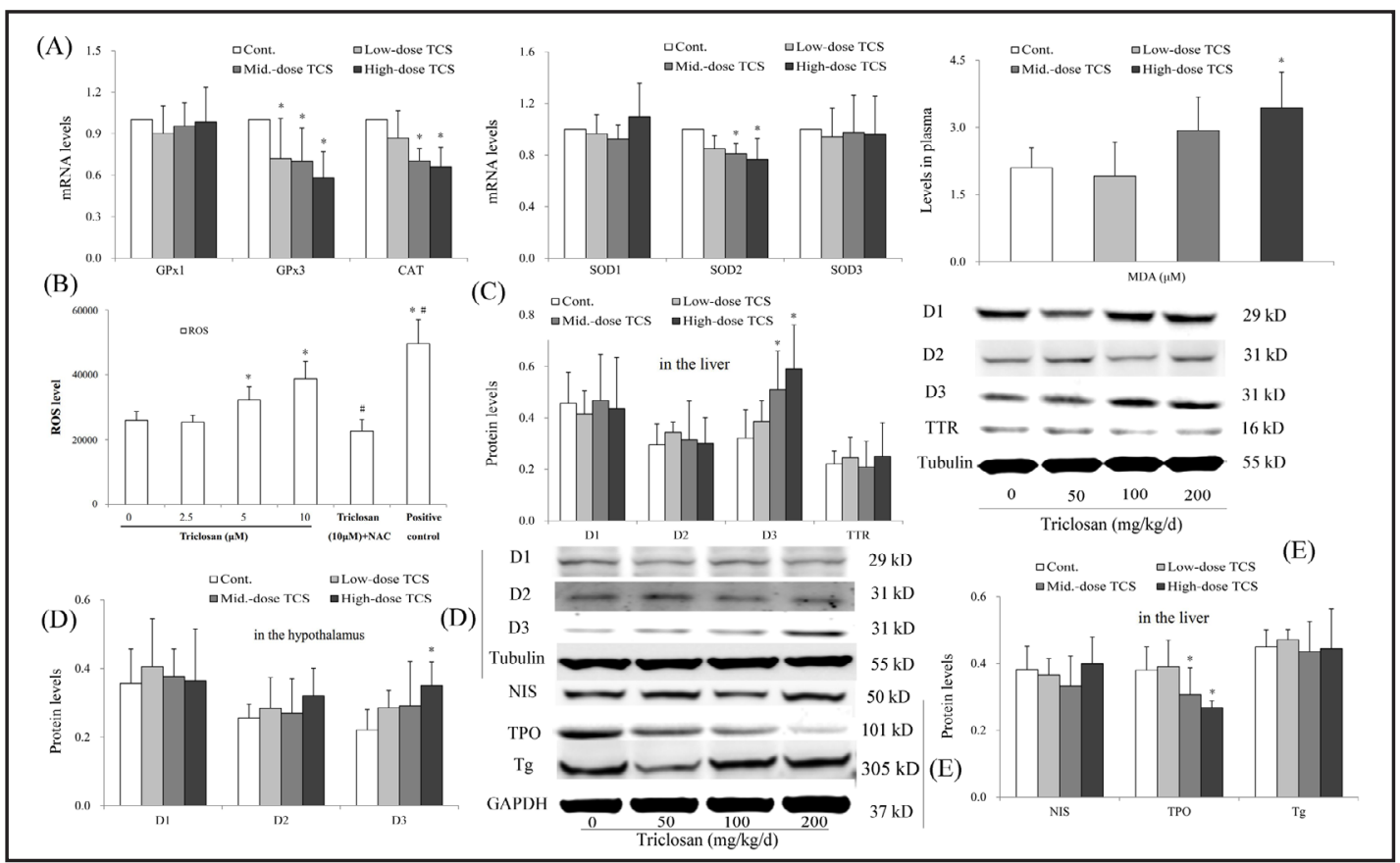

Fig. 2. Effects of triclosan on oxidative stress, ROS production, deiodinases, biotransport and biosynthesis of thyroid hormones. (A) Antioxidant enzymes (Gpx3, CAT, and SOD2) in rat hypothalamus were depleted, whereas MDA was accumulated after triclosan exposure. (B) ROS production in Nthy-ori 3-1 cells was stimulated by triclosan, but was effectively antagonized by NAC. (C) Triclosan induced deiodinase 3 protein expressions in rat liver, but had little interfering effects on the transport protein of THs, TTR. (D) Deiodinase 3 protein level was also upregulated in rat hypothalamus. (E) TPO protein level in rat liver was downregulated by triclosan. ${ }^{*} \mathrm{P}<0.05$, compared with the control. ${ }^{\#} \mathrm{P}<0.05$, compared with the triclosan-treated group. 


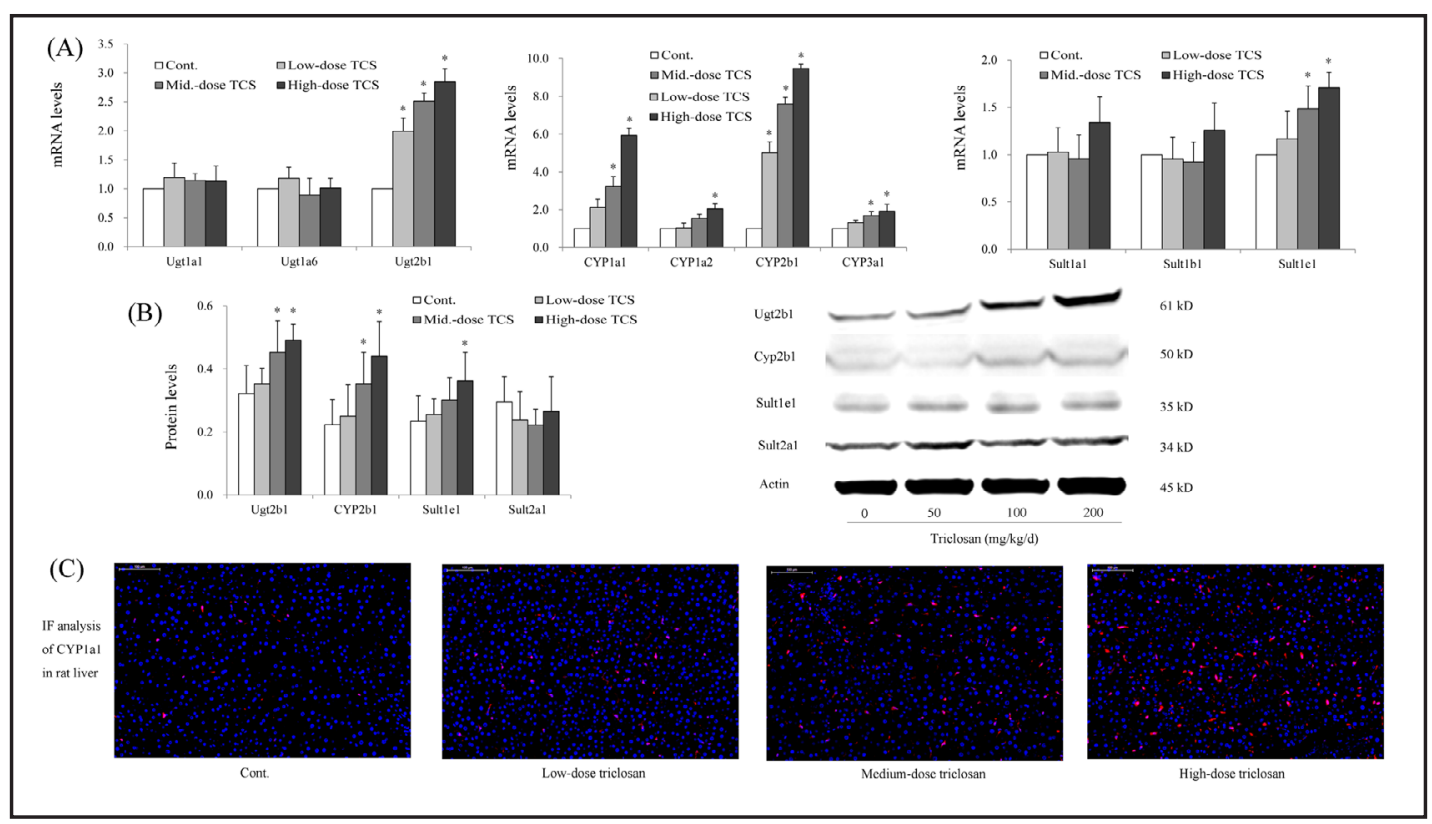

Fig. 3. Effects of triclosan on hepatic enzymes. (A) mRNA expressions of hepatic enzymes (Ugt2b1, CYP1a1, CYP1a2, CYP2b1, CYP3a1, and Sult1e1) in rat liver were induced by triclosan. (B) Triclosan elevated protein levels of hepatic enzymes in rat liver. (C) IF analysis of CYP1a1 in rat liver. Red fluorescence indicated positive expressions of CYP1a1. Magnification: $\times 200 .{ }^{*} \mathrm{P}<0.05$, compared with the control.

mRNA and protein levels in the liver were elevated by $185.2 \%(P<0.001)$ and $53.0 \%(P$ $=0.004$ ), respectively (Fig. 3A, B). Triclosan had stronger inductive effects towards CYPs (CYP1a1, CYP1a2, CYP2b1, and CYP3a1), with the maximum effect observed in CYP2b1. Compared with the control, a 6.6- $(P<0.001)$ and 8.5 -fold $(P<0.001)$ increase in CYP2b1 gene expression was observed in the medium- and high-dose group, respectively (Fig. 3A). CYP2b1 protein level in rat liver exhibited the similar increasing trend ( $P=0.004$; Fig. 3B). IF analysis also showed that the positive expression of CYP1a1 was augmented in rat liver after triclosan exposure (Fig. 3C). Moreover, Sult1e1 in rat liver was also induced by triclosan, and mRNA and protein levels of Sult1e1 in the high-dose group were increased by $71.0 \%(P<$ $0.001)$ and $54.1 \%(P=0.010)$, respectively.

\section{Triclosan suppressed the viability of Nthy-ori 3-1 cells}

Triclosan exerted a concentration-dependent cytotoxic effect on Nthy-ori 3-1 cells, and a significant loss of cell viability was observed in cells treated with different concentrations of triclosan $(0-160 \mu \mathrm{M})$ for 24 or $48 \mathrm{~h}$. After treatment with $10 \mu \mathrm{M}$ triclosan for 24 and $48 \mathrm{~h}$, the cell viability was reduced to $85.5 \%(P=0.008)$ and $80.0 \%(P=0.002)$, respectively (Fig. $4 \mathrm{~A})$.

\section{Triclosan caused histopathologic changes in rat thyroids}

HE staining analysis of rat thyroids revealed histological alterations after triclosan exposure. Thyroids of treated rats exhibited signs of hyperactivity, as indicated by an increase in the number of thyroid follicular epithelial cells $(P<0.001)$ and a decrease in the size of thyroid follicular cavities $(P=0.001)$. Moreover, the cytoplasm that appeared foamy and vacuolated was also observed in the enlarged follicular epithelial cells (Fig. 4B,C). Additionally, triclosan-caused ultrastructural changes were detected by TEM analysis. In the control, normal cell nucleus, chromatin, mitochondria, and rough endoplasmic reticulum were observed. After triclosan exposure, however, the nucleus was deformed, the nuclear membrane was shrinked and chromatin was condensed. Swollen mitochondria, dilated rough endoplasmic reticulum as well as multiple vacuoles were also observed (Fig. 4D).

\section{KARGER}




\section{$\begin{array}{ll}\text { Cellular Physiology } & \text { Cell Physiol Biochem 2018;45:1303-1315 } \\ \text { DOI: 10.1159/000487558 } 102018 \text { The Author(s). Published by S. Karger AG, Basel }\end{array}$

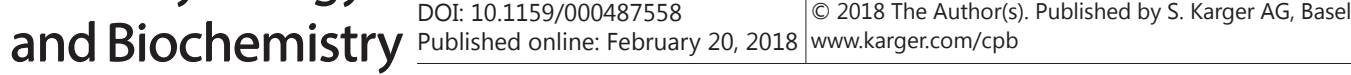

Triclosan activated the JNK and $p 38$ pathways in vivo

After triclosan exposure, the JNK and p38 pathways were activated in rat hypothalamus, dose-dependent increases in p-JNK and p-p38 levels being observed. Compared with the control, p-JNK and p-p38 protein levels in the high-dose group were upregulated by $40.2 \%(P$ $=0.01)$ and $70.3 \%(P=0.006)$, respectively (Fig. $5 \mathrm{~A})$. IF analysis of $\mathrm{p}$-p38 protein expression in the thyroid also suggested that the p38 pathway was activated in vivo, especially in the high-dose group. However, no activated signs of the ERK pathway were observed after triclosan treatment.

\section{Triclosan induced the p38 pathway in vitro}

The p38 pathway was also induced in Nthy-ori 3-1 cells treated with triclosan, and p-p38 protein levels exhibited a concentration-dependent increase. After treatment with 5 and $10 \mu \mathrm{M}$ triclosan for $24 \mathrm{~h}$, a $1.0-(P=0.011)$ and 1.9 -fold $(P<0.001)$ upregulation over the control was noticed (Fig. 5B). However, the time-effect relationship was not observed and only exposure to $10 \mu \mathrm{M}$ triclosan for $24 \mathrm{~h}$, p-p38 protein level was elevated compared with the control $(P<0.001$; Fig. 5C). Intriguingly, the JNK pathway was not activated in vitro and the concentration-effect and time-effect relationships were absent from the current study.

\section{Triclosan activated the p38 pathway via ROS}

To verify the relationship between oxidative stress and the activated p38 pathway, NAC was incubated with triclosan in Nthy-ori 3-1 cells, results indicating that p-p38 protein level was downregulated while ROS production was antagonized by NAC. Compared with the triclosan-treated group, p-p38 protein level was attenuated by $36.4 \%(P=0.012)$ in the combined group (triclosan + NAC) (Fig. 5D).

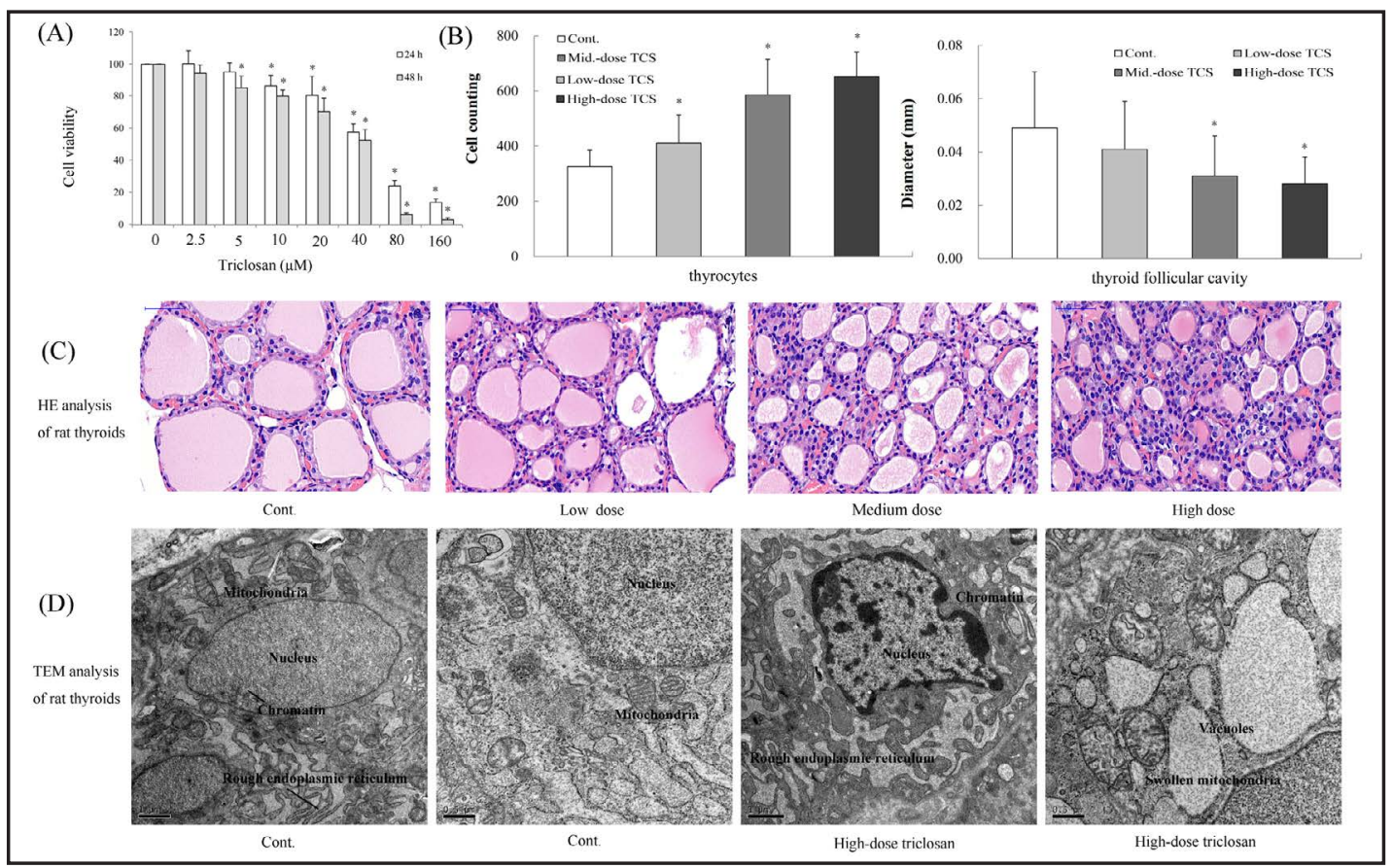

Fig. 4. Effects of triclosan on the cell viability, and histological and ultrastructural changes in the thyroid. (A) Triclosan suppressed the viability of Nthy-ori 3-1 cells. (B,C) Histological changes in the thyroid were quantitatively analyzed by HE staining, as characterized by increased numbers of thyroid follicular epithelial cells and decreased diameters of thyroid follicular cavities. Magnification: $\times 400$. (D) Ultrastructural changes in the thyroid were evaluated by TEM analysis. Magnification: $\times 10000$ and $\times 20000$. ${ }^{*} \mathrm{P}<0.05$, compared with the control. 


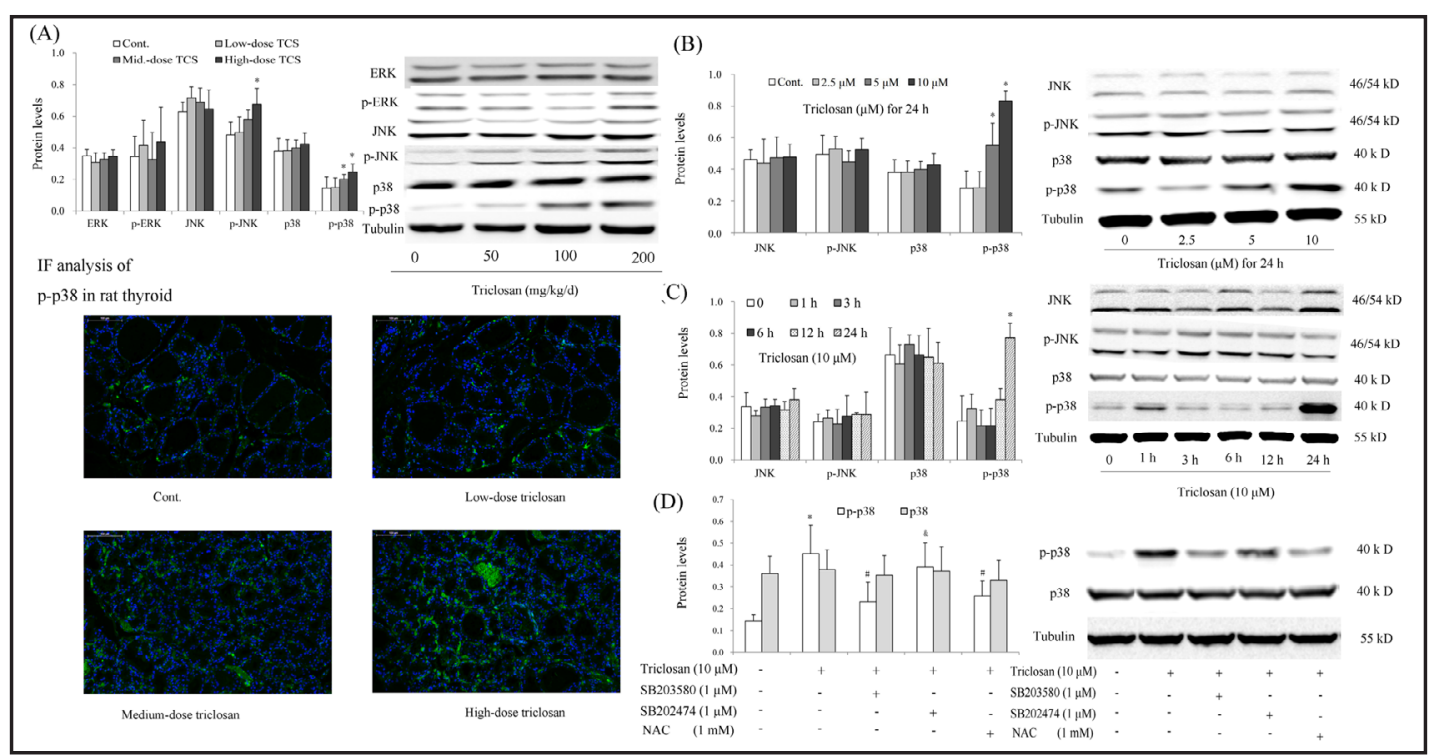

Fig. 5. Effects of triclosan on the MAPK pathway in vivo and in vitro. (A) The p38 and JNK pathways were activated in rat hypothalamus. p-p38 protein expression in rat thyroid was evaluated using IF analysis. Green fluorescence indicated positive expressions. Magnification: $\times 200$. (B) p-p38 protein expression in Nthy-ori 3-1 cells exhibited a concentration-dependent increase after triclosan treatment. (C) After treatment with triclosan for $24 \mathrm{~h}$, the maximum activated effect on p-p38 was observed in Nthy-ori 3-1 cells. (D) The antioxidant (NAC) or the specific inhibitor of p38 (SB203580) could partially but significantly suppress the phosphorylation of p38 in Nthy-ori 3-1 cells. ${ }^{*} \mathrm{P}<0.05$, compared with the control. ${ }^{*} \mathrm{P}<0.05$, compared with the triclosan-treated group. ${ }^{\&} \mathrm{P}<0.05$, compared with the group treated with triclosan and SB203580.

\section{Triclosan induced TRHr expression via the p38 pathway}

To elucidate correlations between the p38 pathway and TR 31 and TRHr, SB203580 and SB202474 were used with triclosan in Nthy-ori 3-1 cells, results demonstrating that SB203580 could partially but significantly inhibited the phosphorylation of p38 (Fig. 5D). After triclosan treatment, TRHr protein level was upregulated in cells; while the p38 pathway was suppressed by SB203580, TRHr protein expression was attenuated by $40.8 \%(P=0.001)$ compared with the triclosan-treated group (Fig. 6A). The decreasing trend of TRHr was also observed in Nthy-ori 3-1 cells pretreated with SB203580 by IF analysis (Fig. 6B). However, TR $\beta 1$ was not induced by triclosan in vitro, and the significant correlation between the p38 pathway and TR $\beta 1$ was absent from the current study.

\section{Induced TRHr contributed to decrements in TPO}

To illuminate the connection between TRHr and TPO, knock down of TRHr gene in Nthy-ori 3-1 cells was performed using TRHr siRNA. After treatment with $400 \mathrm{nM}$ TRHr siRNA for $24 \mathrm{~h}$, TRHr protein level in cells was suppressed by $52.5 \%$ compared with the control $(P<0.001)$, whereas the NC siRNA had little effects on TRHr protein expression (Fig. 6C). Moreover, TPO protein level was attenuated by 38.9\% $(P=0.009)$ in Nthy-ori 3-1 cells exposed to triclosan; however, it was partially but significantly recovered in transfected cells using TRHr siRNA ( $P=0.015$; Fig. 6D). CLSM analysis of TPO also displayed the similar changing characteristics in untransfected and transfected cells (Fig. 6E).

\section{Discussion}

The current work explored the disturbing effects of triclosan on the thyroid and results indicate that triclosan exposure leads to histopathologic changes in the thyroid and p38/ 


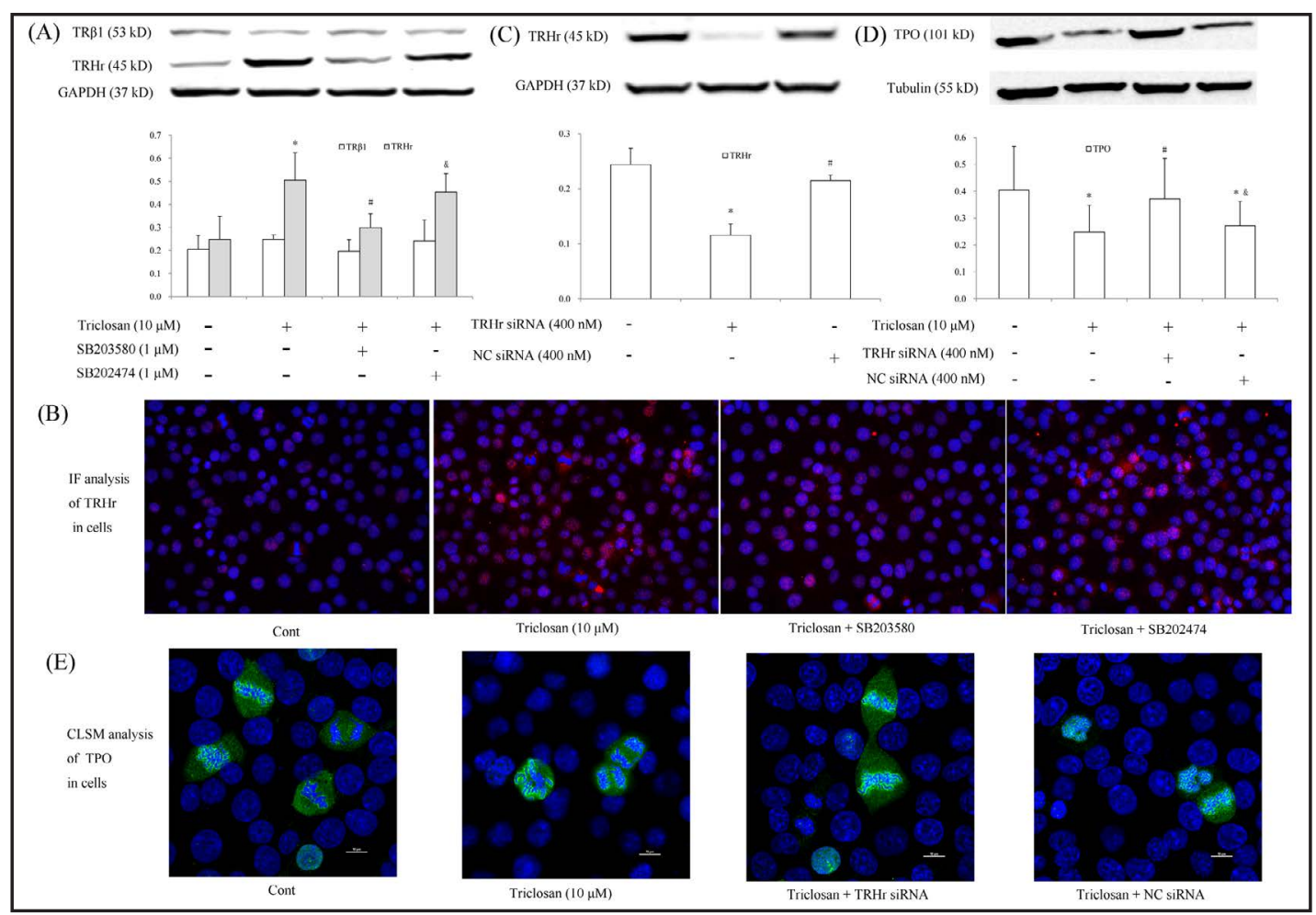

Fig. 6. Effects of triclosan on TR $\beta 1$, TRHr, and TPO in Nthy-ori 3-1 cells. (A) TRHr protein level was increased in Nthy-ori 3-1 cells treated with triclosan, and depressed while the p38 pathway was inhibited by SB203580. (B) TRHr expression was assessed by IF analysis in Nthy-ori 3-1 cells, and red fluorescence indicated positive expressions. Magnification: $\times 400$. (C) TRHr expression was effectively knocked down using TRHr siRNA in Nthy-ori 3-1 cells. (D) TPO was inhibited by triclosan in Nthy-ori 3-1 cells, but it was partially and significantly recovered in transfected cells. (E) CLSM analysis of TPO expression in untransfected or transfected Nthy-ori 3-1 cells. Magnification: $\times 1200{ }^{*} \mathrm{P}<0.05$, compared with the control. ${ }^{\#} \mathrm{P}<0.05$, compared with the triclosan-treated group or the TRHr siRNA-treated group. ${ }^{\circledR} \mathrm{P}<0.05$, compared with the group treated with triclosan and SB203580, or the group treated with triclosan and TRHr siRNA.

TRHr-dependent regulation of TPO plays a vital role in triclosan-induced hypothyroidism. Meanwhile, the disturbance of biotransformation and metabolism of THs also contributes to declines of THs caused by triclosan.

First and foremost, our study proposes a novel mode of action that triclosan decreases THs via ROS/p38/TRHr and mediated TPO. Oxidative stress results from an imbalance between ROS production and neutralization by antioxidants. When ROS production surpasses body's natural antioxidant defense mechanisms, oxidative stress develops and leads to depletions of antioxidant enzymes, lipid peroxidation, DNA damage, etc. After triclosan exposure, mRNA expressions of GPx3, CAT, and SOD2 in rat hypothalamus were suppressed, whereas MDA as a product of polyunsaturated fatty acids peroxidation was accumulated; in vitro study also observed that ROS production was stimulated by triclosan in Nthy-ori 3-1 cells, and these findings are consistent with other studies [15]. It is shown that multiple signal pathways such as the MAPK pathway could be activated and modulated by ROS and oxidative stress [16, 17]. In vivo and in vitro studies, the p38 pathway all displayed activated signs after triclosan exposure. Using the antioxidant NAC further demonstrated the inductive effect of ROS on the p38 pathway, as indicated by decrements in the phosphorylation of p38 after co-incubation with NAC and triclosan in Nthy-ori 3-1 cells. It should be noticed that p-JNK protein level was also elevated in rat hypothalamus; however, the JNK and ERK pathways were not activated in Nthy-ori 3-1 cells treated with triclosan in vitro. We suppose that the p38 pathway, but not the JNK and ERK pathways are involved in triclosan-induced hypothyroidism. However, 


\section{Cellular Physiology Cell Physiol Biochem 2018;45:1303-1315

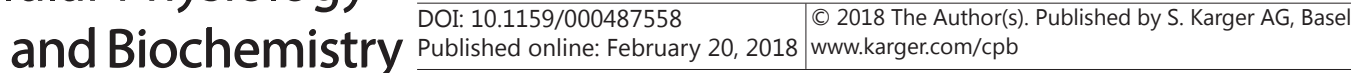 \\ Zhang et al.: Triclosan Causes Hypothyroidism via p38/TRHr-Regulated TPO}

effects of triclosan on the MAPK pathway vary from different studies. In JB6 Cl 41-5a cells, triclosan at $3 \mu \mathrm{M}$ stimulated cell proliferation via activating the ERK and Akt pathways, but not the p38 pathway [18]; in neural stem cells, triclosan at $50 \mu \mathrm{M}$ decreased the cell viability through inducing the phosphorylation of p38 and JNK [15]. We analyze that the diverse effects of triclosan on the MAPK pathway maybe consist in different cells and various concentrations used.

Moreover, our study suggests that TRHr functions significantly in p38 pathway-mediated disturbance of TH homeostasis. TH levels are exquisitely modulated by the HPT axis. THrelated receptors, including TR $\alpha, \mathrm{TR} \beta$, TSHr, and TRHr, are essential elements in regulations of TH synthesis and release. In the HPT axis, the hypothalamus produces TRH that binds to TRHr to stimulate the release of TSH; TSH released by the pituitary binds to TSHr to promote the synthesis and release of THs; THs synthesized by the thyroid interact with TR $\alpha$ and TR $\beta$ to exert pleiotropic effects such as modulating TH homeostasis via the negative feedback system of the HPT axis. In vivo study, TR $\beta 1$ and TRHr protein expressions in rat hypothalamus were upregulated, whereas little changes in TR $\alpha 1$ and TSHr were observed after triclosan exposure; in vitro study, TRHr but not TR $\beta 1$ was still induced after triclosan treatment in Nthy-ori 3-1 cells. To illuminate correlations between the activated p38 pathway and TRHr, the specific inhibitor of p38 was used. When the p38 pathway was activated in vivo and in vitro, upregulated TRHr was observed; while the p38 pathway was inhibited by SB203580 in Nthy-ori 3-1 cells, TRHr protein level was downregulated subsequently. The results indicate that TRHr is modulated by the p38 pathway in the present study. It is shown that abnormal expressions of hormone receptors would interfere with the normal hormone signal transduction in the HPT axis and subsequently disturb TH homeostasis [4]. In our current study, T4 and T3 levels in plasma were reduced and the magnitude of observed hypothyroidism is consistent with previous studies in rats [5, 7], whereas TSH and TRH were not increased as expected to compensate decrements in THs, suggesting impairments of the negative feedback system of the HPT axis. The insensitivity of TSH is regards as a significant marker of the HPT axis and TH imbalance, and our observations are in agreement with other studies on similar endocrine disruptors $[6,14]$. It should be noticed that TR $\beta 1$ protein level was not affected in Nthy-ori 3-1 cells treated with triclosan, preliminarily suggesting that TRß1 is not involved in the p38 pathway-mediated disturbance of TH homeostasis.

Furthermore, the current study indicates that TPO is the key target of the ROS/p38/ TRHr pathway. Tg, NIS, and TPO are essential ingredients for TH synthesis. Tg that contains abundant iodine serves as the scaffold for TH synthesis; NIS mediates the active transport of I- into thyroid follicular cells; TPO catalyzes the iodination of Tyr residues on Tg and then couples these iodotyrosine residues to form T4 and T3 [19]. In our present study, TPO was inhibited in rat liver and Nthy-ori 3-1 cells exposed to triclosan, resulting in suppression of iodination of Tyr residues and subsequent decreases in THs. Wu et al [11]. also reported that triclosan suppressed the activity of TPO at $166 \mu \mathrm{M}$ in FRTL-5 cells. To verify correlations between TPO and the ROS/p38/TRHr pathway, knock down of TRHr gene in Nthy-ori 3-1 cells was performed using TRHr siRNA. Our study observed that TPO protein expression in Nthy-ori 3-1 cells was upregulated after transfection with TRHr siRNA. Therefore, results suggest that TPO is regulated by the ROS/p38/TRHr pathway in the present study and involved in triclosan-induced hypothyroidism. However, Tg and NIS were not affected by triclosan in our current study. We analyze that the absence of TSH stimulation may be the possible reason, because iodinated $\mathrm{Tg}$ that is stored in the follicular lumen of thyroids is released in response to specific hormonal stimulation by TSH [20].

Another mechanism for triclosan-caused hypothyroidism is associated with deiodinase 3 (D3). Deiodinases regulate TH biotransformation via selectively removing iodide from T4 and its derivatives, thus activating/inactivating THs. There are three types of deiodinases, including D1, D2, and D3. D1 is capable of both inner (IRD) and outer ring deiodination (ORD); D2 is capable of ORD to initiate the conversion of T4 to T3 and reverse T3 (rT3)

to $3,3^{\prime}$-diiodothyronine $\left(3,3^{\prime}\right.$-T2); D3 is capable of IRD to catalyze the inactivation of T4 to rT3 and T3 to 3, 3'-T2 [21]. In our present study, D3 protein expression was induced by 


\section{Cellular Physiology Cell Physiol Biochem 2018;45:1303-1315

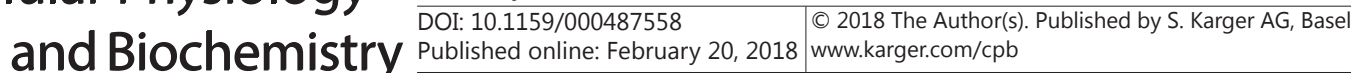 \\ Zhang et al.: Triclosan Causes Hypothyroidism via p38/TRHr-Regulated TPO}

triclosan in rat liver and hypothalamus, catalyzing the conversion of T4 to inactive rT3 and the degradation of T3 to 3, 3'-T2, and eventually leading to declines in T4 and T3. However, no significant changes in D1 and D2 protein levels in the liver and hypothalamus were observed after triclosan exposure. Similar results were also found in other studies [22]. However, the study reported that D2 expression was downregulated in gargarizans embryos that were exposed to 60 and $150 \mu \mathrm{g} / \mathrm{L}$ triclosan [23]. We analyze that differences of research models may explain the diverse effects of triclosan on deiodinases.

In addition, the induction of hepatic enzymes is considered as another dominant mechanism for triclosan-caused hypothyroidism. THs are metabolized predominantly in the liver and excreted into bile. CYPs that are hepatic microsomal phase I enzymes mainly transform and metabolize diverse endogenous compounds such as THs. In our current study, four subtypes of CYPs in rat liver were all induced by triclosan. Moreover, Ugts (hepatic microsomal phase II enzymes) and Sults also contribute to catabolism of THs by increasing the hydrophilicity and biliary and urinary excretion. In our present study, Ugt2b1 and Sult1e1 expressions in the liver were also induced by triclosan. Our findings are consistent with many previous studies, such as the study reported that CYP2B1/2, CYP3A1/23, Ugt1a1, and Sult1c1in the liver were induced after short-term exposure to triclosan [6]. Therefore, another mechanism by which triclosan decreases THs is that triclosan increases metabolism and shortens TH half-life via inducing hepatic enzymes.

\section{Conclusion}

In summary, the present study demonstrates that triclosan has thyroid-disrupting effects. Triclosan exposure leads to declines in THs and histopathologic changes in the thyroid via p38/TRHr and mediated TPO. Meanwhile, our study indicates that induced deiodinase 3 and hepatic enzymes also contribute to hypothyroidism caused by triclosan. Our study would advance a better understanding of the detrimental effects of triclosan on the thyroid.

\section{Acknowledgements}

This work was funded by the Chongqing Population and Family Planning Science and Technology Research Institute (2016cstc-jbky-01701, cstc2017jxjl-scfw0004-01).

\section{Disclosure Statement}

No conflict of interests exists.

\section{References}

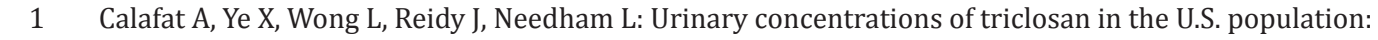
2003-2004. Environ Health Perspect 2008;116:303-307.

-2 Dayan A: Risk assessment of triclosan [Irgasan] in human breast milk. Food Chem Toxicol 2007;45:125129.

3 Dann AB, Hontela A: Triclosan: environmental exposure, toxicity and mechanisms of action. J Appl Toxicol 2011;31:285-311.

4 Ye H, Ha M, Yang M, Yue P, Xie Z, Liu C: Di2-ethylhexyl phthalate disrupts thyroid hormone homeostasis through activating the Ras/Akt/TRHr pathway and inducing hepatic enzymes. Sci Rep 2017;7:40153.

5 Crofton KM, Paul KB, Hedge JM, DeVito MJ: Short-term in vivo exposure to the water contaminant triclosan: Evidence for disruption of thyroxine. Environ Toxicol Pharmacol 2007;24:194-197. 


\section{Cellular Physiology Cell Physiol Biochem 2018;45:1303-1315

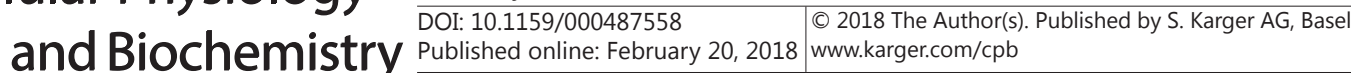

Zhang et al.: Triclosan Causes Hypothyroidism via p38/TRHr-Regulated TPO

6 Paul KB, Hedge JM, DeVito MJ, Crofton KM: Short-term exposure to triclosan decreases thyroxine in vivo via upregulation of hepatic catabolism in young Long-Evans rats. Toxicol Sci 2010;113:367-379.

7 Zorrilla LM, Gibson EK, Jeffay SC, Crofton KM, Setzer WR, Cooper RL, Stoker TE: The effects of triclosan on puberty and thyroid hormones in male Wistar rats. Toxicol Sci 2009;107:56-64.

-8 Pinto PIS, Guerreiro EM, Power DM: Triclosan interferes with the thyroid axis in the zebrafish (Danio rerio). Toxicol Res 2013;2:60-69.

-9 Marlatt VL, Veldhoen N, Lo BP, Bakker D, Rehaume V, Vallée K, Haberl M, Shang D, van Aggelen GC, Skirrow RC, Elphick JR, Helbing CC: Triclosan exposure alters postembryonic development in a Pacific tree frog (Pseudacris regilla) Amphibian Metamorphosis Assay (TREEMA). Aquat Toxicol 2013;126:85-94.

10 Veldhoen N, Skirrow RC, Osachoff H, Wigmore H, Clapson DJ, Gunderson MP, Van Aggelen G, Helbing CC: The bactericidal agent triclosan modulates thyroid hormone-associated gene expression and disrupts postembryonic anuran development. Aquat Toxicol 2006;80:217-227.

11 Wu Y, Beland FA, Fang JL: Effect of triclosan, triclocarban, 2, 2',4 4, 4'-tetrabromodiphenyl ether, and bisphenol A on the iodide uptake, thyroid peroxidase activity, and expression of genes involved in thyroid hormone synthesis. Toxicol In vitro 2016;32:310-319.

12 Hinther A, Bromba CM, Wulff JE, Helbing CC: Effects of triclocarban, triclosan, and methyl triclosan on thyroid hormone action and stress in frog and mammalian culture systems. Environ Sci Technol 2011;45:5395-5402.

13 Sabio G, Cavanagh-Kyros J, Barrett T, Jung DY, Ko HJ, Ong H, Morel C, Mora A, Reilly J, Kim JK, Davis RJ: Role of the hypothalamic-pituitary-thyroid axis in metabolic regulation by JNK1. Genes Dev 2010;24:256-264.

14 Liu C, Ha M, Cui Y, Wang C, Yan M, Fu W, Quan C, Zhou J, Yang K: JNK pathway decreases thyroid hormones via TRH receptor: a novel mechanism for disturbance of thyroid hormone homeostasis by PCB153 Toxicology 2012;302:68-76.

15 Park BK, Gonzales EL, Yang SM, Bang M, Choi CS, Shin CY: Effects of Triclosan on Neural Stem Cell Viability and Survival. Biomol Ther (Seoul) 2016;24:99-107.

16 Ha M, Guan X, Wei L, Li P, Yang M, Liu C: Di-(2-ethylhexyl) phthalate inhibits testosterone level through disturbed hypothalamic-pituitary-testis axis and ERK-mediated $5 \alpha$-Reductase 2 . Sci Total Environ 2016;563-564:566-575.

17 Liu C, Li L, Ha M, Qi S, Duan P, Yang K: The PI3K/Akt and ERK pathways elevate thyroid hormone receptor $\beta 1$ and TRH receptor to decrease thyroid hormones after exposure to PCB153 and p,p'-DDE. Chemosphere 2015;118:229-238.

18 Wu Y, Beland FA, Chen S, Fang JL: Extracellular signal-regulated kinases 1/2 and Akt contribute to triclosanstimulated proliferation of JB6 Cl 41-5a cells. Arch Toxicol 2015;89:1297-1311.

19 Bizhanova A, Kopp P: Minireview: the sodium-iodide symporter NIS and pendrin in iodide homeostasis of the thyroid. Endocrinology 2009;150:1084-1090.

20 Lin JD: Thyroglobulin and human thyroid cancer. Clin Chim Acta 2008;388:15-21.

-21 Gereben B, Zeöld A, Dentice M, Salvatore D, Bianco AC: Activation and inactivation of thyroid hormone by deiodinases: local action with general consequences. Cell Mol Life Sci 2008;65:570-590.

-22 Liu C, Zhao L, Wei L, Li L: DEHP reduces thyroid hormones via interacting with hormone synthesisrelated proteins, deiodinases, transthyretin, receptors, and hepatic enzymes in rats. Environ Sci Pollut Res 2015;22:12711-12719.

23 Chai L, Wang H, Zhao H, Deng H: Chronic effects of triclosan on embryonic development of Chinese toad, Bufo gargarizans. Ecotoxicology 2016;25:1600-1608. 